\title{
Avaliação ecotoxicológica de associações de fármacos utilizados na aquicultura
}

\section{Ecotoxicological assessment of drug combinations used in aquaculture}

\author{
Silvia Patrícia Carraschi ${ }^{1}$, Taise Florêncio ${ }^{1}$, Claudinei da Cruz ${ }^{2}$, Maria José Tavares Ranzani-Paiva ${ }^{3}$ \\ ${ }^{1}$ Centro de Aquicultura, Universidade Estadual Paulista (UNESP) - Jaboticabal (SP), Brasil. \\ ${ }^{2}$ Fundação Educacional de Barretos (UNIFEB) - Barretos (SP), Brasil. \\ ${ }^{3}$ Instituto de Pesca - São Paulo (SP), Brasil.
}

\begin{abstract}
Resumo
Objetivou-se avaliar a interatividade das associações de florfenicol (FFC) + tiametoxam (TH) e enrofloxacino (ENRO) + toltrazurila (TOL) para os seguintes bioindicadores: os peixes pacu (Piaractus mesopotamicus) e mato-grosso (Hyphessobrycon eques), o caramujo (Pomacea canaliculata), a macrófita aquática (Lemna minor) e o microcrustáceo (Daphnia magna). Para tanto, os organismos foram aclimatados em sala de bioensaio, submetidos, inicialmente, a testes de sensibilidade e, nos ensaios definitivos, expostos a concentrações nominais dos fármacos em sistema estático. Para avaliar a toxicidade das associações, utilizaram-se a concentração letal de 50\% (CL50) de cada fármaco isolado e a concentração de cada fármaco responsável pela CL50 da associação, para cada organismo- teste. A associação de TH + FFC apresentou toxicidades antagônica para pacu, mato-grosso, macrófita e caramujo e sinérgica para microcrustáceo. TOL + ENRO foi sinérgica para pacu, macrófita, caramujo, microcrustáceo e antagônica para mato-grosso. TOL + ENRO deve ser utilizada com cautela, pois pode interagir sinergicamente e afetar vários bioindicadores.
\end{abstract}

Palavras-chave: bioindicador; parasiticida; ecotoxicologia; antibiótico.

\begin{abstract}
The aim of this research was to evaluate the interactivity of the combinations florfenicol (FFC) + thiamethoxan (TH) and enrofloxacine (ENRO) + toltrazuril (TOL) for two fish species, pacu (Piaractus mesopotamicus) and mato grosso (Hyphessobrycon eques), a snail (Pomacea canaliculata), a duckweed (Lemna minor) and a microcrustacean (Daphnia magna), which are bioindicators. For this, organisms were first acclimated in a bioassay room and then exposed to the abovementioned substances to verify their sensitivity and after that, in the definitive tests, they were exposed to nominal drug concentrations, in static system. Toxicity of each combination was evaluated using the lethal concentration of 50\% (LC50) of each pure drug and the concentration of each drug responsible for LC50 of the combination, for each organism. The TH + FFC combination showed antagonistic toxicity for the pacu, the mato grosso, the duckweed and the snail but it was synergistic for the microcrustacean. The TOL + ENRO combination was synergistic for the pacu, the duckweed, the snail and the microcrustacean but it was antagonistic for the mato grosso fish. TOL + ENRO must be used with caution because this can synergistically interact and affect several bioindicators.
\end{abstract}

Keywords: bioindicator; parasiticide; ecotoxicology; antibiotic.

Autor para correspondência: Silvia Patrícia Carraschi - Faculdade de Ciências Agrárias e Veterinárias, UNESP - Via de Ac. Prof. Dr. Paulo Donato Castellane, s/n - Zona Rural CEP: 14884-900 - Jaboticabal (SP), Brasil - E-mail: patycarraschi@gmail.com

Recebido em: 11 de fevereiro de 2016

Aceito para publicação em: 30 de setembro de 2016 


\section{Introdução}

A utilização de fármacos na aquicultura tem se tornado uma prática comum a cada ciclo de produção. Tal fato decorre do surgimento de novos patógenos introduzidos por espécies exóticas, do desenvolvimento de resistência e da intensificação dos sistemas de produção (CARRASCHI et al., 2015).

A associação de fármacos, antibióticos e parasiticidas é utilizada na aquicultura, pois, quando uma doença se instala, vários patógenos oportunistas (bactérias e parasitas) acometem o plantel e somente um tipo de fármaco não é suficiente para conter o surto. Os fármacos mais utilizados são antibióticos, como tetraciclinas e fenicóis, e parasiticidas, como organofosforados, metais pesados e formol (SELJESTOKKEN et al., 2006; GUIMARÃES et al., 2007; ROWLAND et al., 2008). Porém, não há estudos sobre a ecotoxicologia e a interação toxicológica que esses fármacos podem causar no ambiente e/ou para o peixe de criação.

Os estudos ecotoxicológicos demonstram a necessidade de uma visão abrangente da toxicidade dos agentes químicos sobre espécies não alvos, pois, levando-se em conta a complexa biota aquática presente no ambiente natural e os diversos graus de sensibilidade entre as espécies, grupos pouco impactados pelo agente podem ser diretamente dependentes de outros que possuem elevada sensibilidade (PRESTES et al., 2013). A utilização de substâncias associadas tem como vantagem o amplo espectro de ação que beneficia o controle de diversos patógenos em um único momento. Com base nos valores de CL50 referentes aos compostos individuais e nos de CL50 referentes a esses compostos na mistura, é possível classificar a magnitude do efeito das misturas para os organismos, sinérgico, antagônico ou aditivo, quando comparada com as concentrações individuais dos componentes da mistura (ZAGATTO e BERTOLETTI, 2006).

O florfenicol (FFC) é um antibiótico do grupo dos fenicóis utilizado em vários países no controle de furunculose, columnariose (INGLIS et al., 1991; USOFR, 2015) e aeromonose (GAIKOWSKI et al., 2003). A sua classificação como praticamente não tóxico para vários organismos, como Onchorynchus mykiss, Lepomis macrochirus, Daphnia magna e Artemia parthenogenetica, foi a principal característica que viabilizou seu estudo como fármaco na aquicultura (FERREIRA et al., 2007;
SCHERING-PLOUGH ANIMAL HEALTH, 2009). O enrofloxacino (ENRO) pertence ao grupo das quinolonas utilizadas no controle de várias bactérias oportunistas (INTORRE et al., 2000; HSU, 2007). Em relação à sua toxicidade, é altamente tóxico para algas e plantas aquáticas (CL50 > 0,1 mg. $\mathrm{L}^{-1}$ ), praticamente não tóxico para microcrustáceos (CL50 > 100 g.L L $^{-1}$ ) (FARMA BASE SAÚDE ANIMAL, 2011) e pouco invasivo para peixes (ROBINSON et al., 2005).

A toltrazurila (TOL) é um parasiticida eficaz no controle de I. multifiliis, um microsporídeo Myxozoa, Trichodina spp. (MEHLHORN et al., 1988; SCHMAHL et al., 1989) e monogenéticos (SCHMAHL e MEHLHORN, 1988). Os dados sobre a toxicidade desse fármaco o descrevem como altamente tóxico para $O$. mykiss $\left(\mathrm{CL} 50<1,0 \mathrm{mg} . \mathrm{L}^{-1}\right)$ e moderadamente tóxico para D. magna (CL50 > 1,0 mg.L ${ }^{-1}$ ) (BAYER HEALTHCARE, 2006).

O tiametoxam (TH) é utilizado como inseticida, mas foi escolhido para ser prospectado no controle de Anacanthorus penilabiatus em pacu. Esse neonicotinoide é praticamente não tóxico para peixes, microcrustáceos e algas (CL50 > $100 \mathrm{mg} . \mathrm{L}^{-1}$ ) (NOVARTIS, 2001; SYNGENTA, 2006).

O parasiticida é indicado para ser administrado pela manhã, na forma de banho terapêutico, e o antibiótico, adicionado à ração, ofertado no período da tarde. Assim, é importante que o efluente dos tanques seja tratado antes do seu descarte para o ambiente, para não causar impacto negativo nos organismos não alvos (CARRASCHI et al., 2014).

Assim, neste estudo, objetivou-se avaliar a interação toxicológica das associações de TH + FFC e ENRO + TOL para os seguintes bioindicadores: peixes pacu (Piaractus mesopotamicus) e mato-grosso (Hyphessobrycon eques), o caramujo de água doce (Pomacea canaliculata), a macrófita aquática Lemna minor e o microcrustáceo Daphnia magna.

\section{Material e Métodos}

Para obtenção dos fármacos, foram utilizadas as seguintes formulações comerciais: Baycox ${ }^{\circledR}(2,5 \mathrm{~g}$ de TOL. $\left.100 \mathrm{~mL}^{-1}\right)$ e Baytril ${ }^{\circledR}\left(10 \mathrm{~g}\right.$ de ENRO. $\left.100 \mathrm{~mL}^{-1}\right)$, fornecidos pela Bayer ${ }^{\odot}$ HealthCare; Agita ${ }^{\circledR} 10 \mathrm{WG}$ (10 g de THI. $100 \mathrm{~g}^{-1}$ ) (Novartis ${ }^{\odot}$ Animal Health), adquirido de forma comercial, e Aquaflor ${ }^{\circledR}(500 \mathrm{~g}$ de FFC. $\mathrm{kg}^{-1}$ ), fornecido pela MSD ${ }^{\odot}$.

Os procedimentos com os animais foram enviados e aprovados pelo Comitê de Ética da 
Faculdade de Ciências Agrárias e Veterinárias da Universidade Estadual Paulista, em Jaboticabal (SP), sob o no 017335/10.

Os ensaios com os fármacos individualmente foram publicados por Carraschi et al. (2015) e, a seguir, constam os valores da concentração letal de cada fármaco para cada bioindicador (Tabela 1).

\section{Ensaios de toxicidade aguda para os peixes}

Nos ensaios de toxicidade, utilizaram-se o pacu, com peso entre 0,5 e 1,0 g, e o mato-grosso, entre 0,35 e $0,8 \mathrm{~g}$, aclimatados por dez dias em sala de bioensaio a $25,0 \pm 2,0^{\circ} \mathrm{C}$ e fotoperíodo de 12 horas de luz.

Primeiramente, para avaliar a sensibilidade do lote de organismos-teste, realizou-se ensaio de toxicidade com cloreto de potássio como substância referência (ABNT, 2011), sendo a concentração letal média (CL50; 48 h) de 1,54 g... $\mathrm{L}^{-1}$, com intervalo de confiança de $95 \%$ entre 1,28 e 1,86 g.L $\mathrm{L}^{-1}$ para o pacu e 2,20 g.L. ${ }^{-1}\left(1,84\right.$ a 2,67 g.L. $\left.{ }^{-1}\right)$ para o mato-grosso.

Nos ensaios definitivos da associação de TOL + ENRO, utilizaram-se as seguintes concentrações: $10,0,15,0,20,0$ e 25,0 mg.. $\mathrm{L}^{-1}$ para o pacu e $25,0,35,0,45,0$ e 55,0 mg.L L $^{-1}$ para o mato-grosso; na de TH + FFC, utilizaram-se o controle e $100,0 \mathrm{mg} . \mathrm{L}^{-1}$ para ambos os peixes, com três réplicas, três peixes por réplica, na densidade máxima de 1 g. $\mathrm{L}^{-1}$, em sistema estático com duração de 48 horas (ABNT, 2011).

\section{Ensaios de toxicidade aguda para o caramujo Pomacea canaliculata}

A norma utilizada para o caramujo foi a mesma que para os peixes (ABNT, 2011), com algumas adaptações.

Os caramujos com peso entre $1,0 \mathrm{e} 2,0 \mathrm{~g}$ foram selecionados e aclimatados em sala de bioensaio a $25 \pm 2{ }^{\circ} \mathrm{C}$, em fotoperíodo de 12 horas de luz e alimentados diariamente com Hydrilla verticillata (VENTURINI et al., 2008).
Nos ensaios de sensibilidade com cloreto de potássio, a concentração efetiva média (CE50;48 h) foi de 1,49 g. $\mathrm{L}^{-1}$, com intervalo de confiança de $95 \%$ entre 1,14 g.L $\mathrm{L}^{-1}$ e 1,96 g.L $\mathrm{L}^{-1}$.

Nos ensaios definitivos, utilizaram-se as seguintes concentrações de TOL + ENRO: 1,0, 10,0, 25,0, 50,0 e 100,0 mg.L $\mathrm{L}^{-1}$ e de TH + FFC: $60,0,70,0,80,0,90,0$ e 100,0 mg. $\mathrm{L}^{-1}$ e um controle. O ensaio apresentou duração de 48 horas, em sistema estático, com cinco caramujos por réplica e três réplicas cada concentração. A avaliação da mortalidade foi realizada por meio de imobilidade durante dez segundos de estímulo.

\section{Toxicidade aguda para a macrófita aquática Lemna minor}

As plantas foram cultivadas em casa de vegetação, em caixas de $1 \mathrm{Le}$, após seu crescimento, transferidas para cristalizadores em meio de cultivo (Hoagland 's) em sala de bioensaio com temperatura de $25 \pm 2{ }^{\circ} \mathrm{C}$ e fotoperíodo de 24 horas de luz, por quatro dias (OECD, 2006).

$\mathrm{Na}$ avaliação da sanidade do lote das plantas, foi realizado um ensaio com a substância referência cloreto de sódio $(\mathrm{NaCl})$, em que a CL50;7d média foi de $6,67 \mathrm{~g} . \mathrm{L}^{-1}$, com intervalo de confiança de $95 \%$ entre 5,48 g.L. $\mathrm{L}^{-1}$ e 6,85 g.L $\mathrm{L}^{-1}$. As plantas foram desinfetadas em solução de hipoclorito a $0,5 \%$ e, em seguida, quatro colônias com três frondes $(\mathrm{n}=12)$ foram selecionadas para cada repetição. Nos ensaios definitivos, as plantas foram expostas a $0,1,10,0,20,0,40,0,60,0,80,0$ e 100,0 mg. $\mathrm{L}^{-1}$ de TOL + ENRO e a $0,0,60,0,70,0,80,0,90,0$ e 100,0 mg.L $\mathrm{L}^{-1}$ de TH + FFC.

Os ensaios foram conduzidos em sistema estático, com três réplicas em cada concentração, com duração de sete dias. A letalidade das plantas foi avaliada três, cinco e sete dias após a exposição, tendo se observado aumento do número de frondes, clorose (perda de clorofila) e necrose (morte das plantas).

Tabela 1. Concentração letal (CL/CE) dos fármacos isolados para os bioindicadores (CARRASCHI et al., 2015).

\begin{tabular}{lccccc}
\hline \multirow{2}{*}{ Fármacos } & \multicolumn{5}{c}{ CL/CE50 (mg.L $\mathbf{~}^{-1}$ ) } \\
\cline { 2 - 6 } & $\boldsymbol{P .}$ mesopotamicus & H. eques & $\boldsymbol{P .}$ canaliculata & L. minor & D. magna \\
\hline Florfenicol & $>100$ & $>100$ & $>100$ & 97,03 & $>100$ \\
Enrofloxacino & 116,70 & $>100$ & 14,64 & 60,49 & 84,39 \\
Toltrazurila & 3,72 & 6,22 & 7,59 & $>100$ & 18,57 \\
Tiametoxam & 16,97 & 49,78 & 87,14 & $>100$ & 107,18 \\
\hline
\end{tabular}




\section{Toxicidade aguda para o microcrustáceo Daphnia magna}

Os organismos foram cultivados em cristalizadores, com o meio de cultivo renovado duas vezes por semana, temperatura controlada de $20 \pm 2{ }^{\circ} \mathrm{C}$, fotoperíodo de 16 horas de luz e alimentados por uma suspensão de algas Scenedesmus subspicatus ( $5 \times 10^{6}$ células/indivíduo/dia) e um composto de ração fermentada para peixes e solução de levedura (Saccharomyces cerevisiae) (ABNT, 2009).

A sensibilidade foi avaliada utilizando cloreto de sódio como substância referência, com CE50;48 h média de 4,31 g.L. $\mathrm{L}^{-1}$.

Para os ensaios definitivos, foram utilizadas concentrações de 5,0,10,0,15,0, 20,0, 25,0, 30, 35,0 mg.L $\mathrm{L}^{-1}$ de TOL + ENRO e de 3,0, 5,0, 10,0, 20,0, 30,0, 40,0 mg. $\mathrm{L}^{-1}$ de TH + FFC e um controle. Os ensaios apresentaram cinco neonatos cada réplica, com 4 a 24 horas de vida, quatro réplicas cada concentração, duração de 48 horas, tendo sido mantidos no escuro.

\section{Interatividade das substâncias das associações}

As associações foram compostas de $50 \%$ de cada fármaco e, após a obtenção das CL/CE50 de cada fármaco individualmente e das associações, foi utilizado o modelo descrito por Marking (1977), em que expressa a soma das substâncias (S) e o índice de aditividade (IA), como descritos a seguir:

$\mathrm{S}=(\mathrm{Am} / \mathrm{Ai})+(\mathrm{Bm} / \mathrm{Bi})$

sendo:

$\mathrm{S}=$ soma da aditividade;

$\mathrm{Am}=$ concentração da substância A na associação que causa $50 \%$ de efeito;

$\mathrm{Bm}=$ concentração da substância B na associação que causa $50 \%$ de efeito;

$\mathrm{Ai}=$ concentração da substância A que, isolada, causa $50 \%$ de efeito (CL/CE50);

$\mathrm{Bi}=$ concentração da substância $\mathrm{B}$ que, isolada, causa $50 \%$ de efeito (CL/CE50).
Portanto, pela soma (S), é possível determinar o efeito interativo das substâncias, caracterizado por:

$\mathrm{S}>1$; o efeito da associação é

menos que aditivo (antagônico)

$\mathrm{S}=1 ;$ o efeito é aditivo

$\mathrm{S}<1$; o efeito é mais que aditivo (sinérgico)

Assim, é possível calcular o índice de aditividade (IA) e conhecer quantas vezes a associação é mais ou menos tóxica do que a soma da toxicidade dos fármacos individualmente:

Se $\mathrm{S}>1 \rightarrow \mathrm{IA}=\mathrm{S}(-1)+1$

$\mathrm{Se} \mathrm{S}<1 \rightarrow \mathrm{IA}=1 / \mathrm{S}-1$

\section{Resultados}

\section{Piaractus mesopotamicus}

A CL50;48 h da associação de TOL + ENRO foi de $16,15 \mathrm{mg} . \mathrm{L}^{-1}$, mas com $10,0 \mathrm{mg} . \mathrm{L}^{-1}$ não ocorreu mortalidade, com 15,0 mg. $\mathrm{L}^{-1}, 33,33 \%$, $20,83,33 \%$ e com 25,0 mg. $\mathrm{L}^{-1}, 100 \%$ (Tabela 2 ).

A CL50;48 h da associação de FFC + TH foi maior que $100 \mathrm{mg} . \mathrm{L}^{-1}$, pois não ocorreu mortalidade até $100 \mathrm{mg} \cdot \mathrm{L}^{-1}$, concentração máxima testada (Tabela 2).

\section{Hyphessobrycon eques}

A CL50;48 h da associação de TH + FFC foi maior que $100 \mathrm{mg} . \mathrm{L}^{-1}$, pois não ocorreu mortalidade nas concentrações avaliadas, e a da associação TOL + ENRO foi de 37,92 mg.L $\mathrm{L}^{-1}$, em que no controle e em $25 \mathrm{mg} . \mathrm{L}^{-1}$ não ocorreu mortalidade; em 35,0 e 45,0 mg. $\mathrm{L}^{-1}$, a mortalidade foi de $55,56 \%$ e, em 55 mg. $\mathrm{L}^{-1}, 100 \%$ (Tabela 3 ).

\section{Pomacea canaliculata}

A CE50;48 h da associação de TOL + ENRO foi de $12,34 \mathrm{mg} \cdot \mathrm{L}^{-1}$. No controle, não ocorreu imobilidade, em 1,0 mg. $\mathrm{L}^{-1}$ a imobilidade foi de

Tabela 2. Concentração letal (CL50;48 h) das associações de fármacos para Piaractus mesopotamicus.

\begin{tabular}{lccccc}
\hline \multicolumn{1}{c}{ Fármacos } & Limite inferior & $\begin{array}{c}\text { CL50;48 h } \\
\left(\mathbf{m g . L ^ { - 1 } )}\right.\end{array}$ & Limite superior & Equação linear & $\mathbf{R}^{2}$ \\
\hline TOL+ENRO & 15,35 & 16,15 & 18,22 & $\mathrm{y}=7 \mathrm{x}-68.333$ & 0,97 \\
TH+FFC & - & $>100$ & - & - & - \\
\hline
\end{tabular}

-: não calculado. 
$6,7 \%, 10,0,33,33 \%, 25,0,66,7 \%, 50,0,93,3 \%$ e, em 100 mg. L $^{-1}, 100 \%$ (Tabela 4).

A CE50;48 h da associação de TH + FFC foi superior a $100,0 \mathrm{mg} \cdot \mathrm{L}^{-1}$, pois não ocorreu, no mínimo, 50\% de imobilidade. No controle, não ocorreu imobilidade, em 60,0 mg.L $\mathrm{L}^{-1}$, a imobilidade foi de $13,3 \%, 70,0,20,0 \%, 80,0,13,3 \%, 90,0$, 20,0\% e, em 100 mg. $\mathrm{L}^{-1}, 20 \%$ (Tabela 4).

\section{Lemna minor}

A CL50;7d da associação de TOL + ENRO foi de 7,03 mg.L $\mathrm{L}^{-1}$. No controle, não ocorreu mortalidade, em $0,1 \mathrm{mg} . \mathrm{L}^{-1}$ ocorreu 33\%, 10,0, 51\%, 20,0, 52\%, $40,0,53 \%, 60,60 \%$, 80,0 e, em 100,0 mg.L ${ }^{-1}$, $65 \%$ (Tabela 5).

A CL50;7d da associação TH + FFC foi superior a $100,0 \mathrm{mg} \cdot \mathrm{L}^{-1}$. No controle, não ocorreu mortalidade; em 60,0 mg.L $\mathrm{L}^{-1}$, ocorreu $31 \%, 70,0,37 \%, 80,0,32 \%, 90,0,47 \%$ e, em 100,0 mg.L $\mathrm{L}^{-1}, 49 \%$ (Tabela 5).

\section{Daphnia magna}

A CE50;48 h da associação de TOL + ENRO foi de $23,51 \mathrm{mg} \cdot \mathrm{L}^{-1}$. No controle, não ocorreu imobilidade; em 5,0 e 10,0 mg. $\mathrm{L}^{-1}$, foi de $5,0 \%$, $15,0,15,0 \%, 20,0$ e $15,0,20,0 \%, 30,0,60,0 \%$ e, em 35 mg.L. ${ }^{-1}, 90 \%$ (Tabela 6).

A CE50;48 h da associação de TH + FFC foi de $14,57 \mathrm{mg} . \mathrm{L}^{-1}$. No controle e em $1,0 \mathrm{mg} . \mathrm{L}^{-1}$, não ocorreu imobilidade; em $3,0 \mathrm{mg} . \mathrm{L}^{-1}$, foi de $15,0 \%$, $5,0,20,0 \%, 10,0,25,0 \%, 20,0,40,0 \%, 30,65,0 \%$ e, em 40 mg.L $\mathrm{L}^{-1}, 100 \%$ (Tabela 6).

\section{Interatividade das associações}

A associação de TOL + ENRO apresentou interações sinérgica $(\mathrm{S}<1)$ para $P$. mesopotamicus, L. minor, P. canaliculata e D. magna e antagônica ( $\mathrm{S}>1$ ) para H. eques. A associação de $\mathrm{TH}+\mathrm{FFC}$ foi antagônica $(\mathrm{S}>1)$ para macrófita, caramujo, pacu e mato-grosso e sinérgica $(\mathrm{S}<1)$ para D. magna (Tabela 7).

Tabela 3. Concentração letal (CL50;48 h) das associações de fármacos para Hyphessobrycon eques.

\begin{tabular}{lccccc}
\hline Fármacos & Limite inferior & $\begin{array}{c}\text { CL50;48 h } \\
\left(\mathbf{m g . L ^ { - 1 } )}\right.\end{array}$ & Limite superior & Equação linear & $\mathbf{R}^{2}$ \\
\hline TOL+ENRO & 30,60 & 37,92 & 46,99 & $\mathrm{y}=2,3334 \mathrm{x}-$ & 0,82 \\
TH+FFC & - & $>100$ & - & 46,111 & - \\
\hline
\end{tabular}

- não calculado.

Tabela 4. Concentração estimada (CE50;48 h) das associações de fármacos para Pomacea canaliculata.

\begin{tabular}{lccccc}
\hline Fármacos & Limite superior & $\begin{array}{c}\text { CE50;48 h } \\
\left(\mathbf{m g . L ^ { - 1 } )}\right.\end{array}$ & Limite inferior & Equação linear & $\mathbf{R}^{2}$ \\
\hline TOL+ENRO & 21,10 & 12,34 & 7,22 & $\mathrm{y}=0.87 \mathrm{x}+$ & 0,76 \\
TH+FFC & - & $>100,0$ & - & $\mathrm{y}=0,13 \mathrm{x}+6,6$ & 0,33 \\
\hline
\end{tabular}

Tabela 5. Concentração letal (CL50;48 h) das associações de fármacos para o bioindicador Lemna minor.

\begin{tabular}{lccccc}
\hline \multicolumn{1}{c}{ Fármacos } & Limite superior & $\begin{array}{c}\text { CL50;7d } \\
\left(\mathbf{m g . L ^ { - 1 } )}\right.\end{array}$ & Limite inferior & Equação linear & $\mathbf{R}^{2}$ \\
\hline TOL+ENRO & 20,93 & 7,03 & 2,36 & $\mathrm{y}=1,5925 \mathrm{x}+$ & 0,85 \\
TH+FFC & - & $>100$ & - & $\mathrm{y}=1.5196 \mathrm{x}+$ & 0,77 \\
\end{tabular}

Tabela 6. Concentração estimada (CE50;48 h) das associações de fármacos para o bioindicador Daphnia magna.

\begin{tabular}{lccccc}
\hline \multicolumn{1}{c}{ Fármacos } & Limite superior & $\begin{array}{c}\text { CE50;48 h } \\
\left(\mathbf{m g . L ^ { - 1 } )}\right.\end{array}$ & Limite inferior & Equação linear & $\mathbf{R}^{\mathbf{2}}$ \\
\hline TOL+ENRO & 26,47 & 23,51 & 20,88 & $\mathrm{y}=2,82 \mathrm{x}-$ & 0,93 \\
TH+FFC & 18,97 & 14,57 & 11,34 & $\mathrm{y}=2,25 \mathrm{x}+2,72$ & 0,92 \\
\hline
\end{tabular}


Tabela 7. Avaliação da soma (S) e do índice de aditividade (IA) das associações utilizadas nos ensaios de toxicidade aguda para os organismos-teste.

\begin{tabular}{lccccc}
\hline \multirow{2}{*}{ Organismos-teste } & \multicolumn{2}{c}{ Toltrazurila + enrofloxacino } & & \multicolumn{2}{c}{ Tiametoxam + florfenicol } \\
\cline { 2 - 3 } \cline { 5 - 6 } Piaractus mesopotamicus & S & IA & & S & IA \\
Lemna minor & 0,11 & 0,02 & & 1,81 & $-0,81$ \\
Pomacea canaliculata & 0,99 & 8,43 & & 1,01 & $-0,1$ \\
Daphnia magna & 0,47 & 0,01 & & 1,02 & $-0,2$ \\
Hyphessobrycon eques & 1,52 & 1,12 & & 0,05 & 19,83 \\
\hline
\end{tabular}

Dessa forma, a toxicidade da associação de TOL + ENRO é $0,03,8,43,0,01$ e 1,12 vez maior para pacu, macrófita, caramujo e microcrustáceo, respectivamente, e 0,52 vez menor para mato-grosso do que a toxicidade de cada fármaco isoladamente.

A toxicidade da associação de TH + FFC é de $0,81,0,1,0,22$ e 0,16 vez menor para pacu, macrófita, caramujo e mato-grosso, respectivamente, e 19,83 vezes maior para D. magna do que cada fármaco isoladamente (Tabela 7).

\section{Discussão}

A associação de TH + FFC apresentou efeito ecotoxicológico antagônico $(\mathrm{S}>1)$ para a maioria dos bioindicadores, assim como a mistura de sulfato de cobre e peróxido de hidrogênio para $D$. similis (ZAGATTO e BERTOLETTI, 2006). O efeito antagônico entre os fármacos é importante, uma vez que se utilizados no tratamento de doenças na aquicultura, não causarão toxicidade aos organismos não alvos. Nesse caso, para D. magna, o efeito foi sinérgico, com fator de magnificação de toxicidade 19,8 vezes maior em mistura, sendo esse organismo muito sensível a essa associação. Esse microcrustáceo é modelo para estudos ecotoxicológicos de fármacos em razão de sua sensibilidade e importância na cadeia trófica no ambiente de água doce (CONSTANTINE e HUGGETT, 2010).

A associação de TOL + ENRO foi caracterizada por efeito sinérgico para a maioria dos bioindicadores, sendo mais expressiva para $L$. minor, 8,4 vezes, seguido de D. magna, 1,1 vez maior. Da mesma forma, a associação dos agrotóxicos; diclorvós e deltametrina, para Danio rerio e Hyphessobrycon bifasciatus (TREVIS et al., 2010); dos fungicidas epoxiconazol e piraclostrobin foi 13,6 vezes mais tóxica para a alga Pseudokirchneriella subcapitata (PRESTES e JONSSON, 2011) e 1,17 vez para o tambaqui (Colossoma macropomum)
(PRESTES et al., 2013). O sinergismo evidencia que esses fármacos necessitam de cautela ao serem utilizados na utilização, pois os organismos não alvos podem sofrer um efeito negativo dessas associações no ambiente.

A toxicidade de um xenobiótico varia com a espécie, a idade dos indivíduos, o peso e o nível trófico (MALLATT, 1985). Assim, a toxicidade de cada associação para cada organismo-teste encontrada neste estudo está relacionada com essas variáveis, visto que foram utilizadas diferentes espécies representantes de vários níveis tróficos.

Caso os fármacos sejam utilizados na concentração, tempo de exposição, dias de tratamento e da forma correta (ração ou banho), o efeito dessas associações não deverá oferecer risco aos bioindicadores nem ao ambiente. A interação das substâncias pode ocorrer na fase de exposição (aguda e crônica), toxicocinética (absorção, biotransformação, distribuição, armazenamento e excreção) ou de toxicodinâmica (nos sítios de ação) (ZAGATTO e BERTOLETTI, 2006).

A TOL é um derivado da triazinetriona e causa redução das enzimas da cadeia respiratória dos parasitas e inibição da divisão nuclear (EMEA, 2008) e o enrofloxacino (ENRO) é um antibiótico do grupo das fluoroquinolonas que inibe a atividade da DNA girase (KOC et al., 2009). O florfenicol (FFC) é um antibiótico fluorado derivado de tianfenicol, inibidor da síntese de proteína (CANNON et al., 1990), e o tiametoxam (TH) é um inseticida neonicotinoide, agonista dos receptores nicotínicos (TOMIZAWA e CASIDA, 2001). Dessa forma, devem ser desenvolvidos estudos para conhecer como essas substâncias interagem com o ambiente e manifestam antagonismo ou sinergismo a este.

A avaliação do uso integrado dos fármacos na aquicultura está em fase inicial, porém se mostra cada vez mais necessária à medida que se torna imprescindível o uso de mais de um fármaco para evitar a dizimação de plantéis acometidos por 
surtos de doenças. Assim, essa avaliação deverse-á intensificar à medida que a prática se tornar mais frequente.

\section{Conclusão}

A utilização do parasiticida toltrazurila e do antibiótico enrofloxacino em um mesmo lote de peixes deve ser cautelosa, uma vez que podem interagir quimicamente no ambiente e manifestar toxicidade sinérgica aos organismos não alvos. A utilização de tiametoxam e florfenicol apresenta antagonismo, porém exerce efeito sinérgico para D. magna, um bioindicador mais sensível.

\section{Agradecimentos}

À Fapesp, Fundação de Amparo à Pesquisa do Estado de São Paulo, pela bolsa processo no ${ }^{\circ}$ 2008/51900-3 e auxílio concedidos no 2011/08453-9.

\section{Referências}

ASSOCIAÇÃO BRASILEIRA DE NORMAS TÉCNICAS - ABNT. NBR 12713: ecotoxicologia aquática: toxicidade aguda: método de ensaio com Daphnia ssp (Crustacea, Cladocera). 3. ed. São Paulo, 2009. 22 p.

ASSOCIAÇÃO BRASILEIRA DE NORMAS TÉCNICAS - ABNT. NBR 15088: ecotoxicologia aquática: toxicidade aguda: método de ensaio com peixes. São Paulo, 2011. 19 p.

BAYER HEALTHCARE. Ficha de Informações de Segurança de Produto Químico (FISPQ): Baycox oral. 2006. 5 p.

CANNON, M.; HARFORD, S.; DAVIES, J. A comparative study on the inhibitory actions of chloramphenicol, thiamphenicol and some fluorinated derivatives. The Journal of Antimicrobial Chemotherapy, v. 2, p. 307-317, 1990.

CARRASCHI, S.P.; BARBUIO, R.; IKEFUTI, C.V.; FLORÊNCIO, T.; CRUZ, C.; RANZANIPAIVA, M.J.T. Effectiveness of therapeutic agents in disease treatment in Piaractus mesopotamicus. Aquaculture, v. 431, p. 124-128, 2014.

CARRASCHI, S.P.; FLORENCIO, T.; GARLICH, N.; SILVA, A.F.; MARQUES, A.M.; CRUZ, C.; PAIVA, M.J.T.R. Ecotoxicology of drugs used in fish disease treatment. Journal of Environmental
Chemistry and Ecotoxicology, v. 7, n. 3, p. 3136, 2015.

CONSTANTINE, L.A.; HUGGETT, D.B. A comparison of the chronic effects of human pharmaceuticals on two cladocerans, Daphnia magna and Ceriodaphnia dubia. Chemosphere, v. 80, n. 9, p. 1069-1074, 2010.

EUROPEAN MEDICINES AGENCY - EMEA. Anexo I: lista das denominações, forma farmacêutica, dosagem dos medicamentos veterinários, espécies animais, via(s) de administração, titulares das autorizações de introdução no mercado nos estados-membros. London, 2008. 10 p.

FARMA BASE SAÚDE ANIMAL. Ficha de Informação de Segurança de Produtos Químicos (FISPQ): Eurofarm 5\%. 2011. 5 p.

FERREIRA, C.S.G.; NUNES, B.A.; HENRIQUESALMEIDA, J.M.M.; GUILHERMINO, L. Acute toxicity of oxytetracycline and florfenicol to the microalgae Tetraselmis chuii and to the crustacean Artemia parthenogenetica. Ecotoxicology and Environmental Safety, v. 67, p. 452-458, 2007.

GAIKOWSKI, M.P.; WOLF, J.C.; ENDRIS, R.G. Gingerich WHSafety of Aquaflor (Florfenicol, 50\% Type A Medicated Article), Administered in Feed to Channel Catfish, Ictalurus punctatus. Toxicologic Pathology, v. 3, p. 689-697, 2003.

GUIMARÃES, A.T.B.; ASSIS, H.S.; BOEGER, W. The effect of trichlorfon on acetylcholinesterase activity and histopathology of cultivated fish Oreochromis niloticus. Ecotoxicology and Environmental Safety, v. 68, n. 1, p. 57-62, 2007.

HSU, H.M. Bowser PR, Schachte JH, Scarlett JMJR, Babish JG.Winter Winter Field Trials of Enrofloxacin for the Control of Aeromonas salmonicida Infection in Salmonids. Journal of the World Aquaculture Society, v. 26, n. 3, p. 307-314, 2007.

INGLIS, V.; RICHARDS, R.H.; VARMA, K.J.; SUTHERLAND, I.H.; BROKKEN, E.S. Florfenicol in Atlantic salmon, Salmo salar L, parr: tolerance and assessment of efficacy against furunculosis. Journal of Fish Diseases, v. 14, p. 343-351, 1991. 
INTORRE, L.; CECCHINI, S.; BERTINI, S.; VARRIALE, A.M.C.; SOLDANI, G.; MENGOZZI, G. Pharmacokinetics of enrofloxacin in the seabass Dicentrarchus labrax. Aquaculture, v. 182, p. 49-59, 2000.

KOC, F.; UNEY, K.; ATAMANALP, M.; TUMER, I.; KABAN, G. Pharmacokinetic disposition of enrofloxacin in brown trout (Salmo trutta fario) after oral and intravenous administrations. Aquaculture, v. 295, n. 1, p. 142-144, 2009.

MALLATT, J. Fish gill structural changes induced by toxicants and other irritants: a statistical review. Canadian Journal of Fisheries and Aquatic Sciences, v. 42, p. 630-648, 1985.

MARKING, L.L. Method for assessing additive toxicity of chemical mistures. In: MAYER, F.L.; HAMELINK, J. (editores). Aquatic toxicology and hazard evaluation: ASTM STP 634. 1977. p. 99-108.

MEHLHORN, H.; SCHMAHL, G.; HABERKORN, A. Toltrazuril effective against a broad spectrum of protozoan parasites. Short communication. Parasitology Research, v. 75, p. 64-66, 1988.

NOVARTIS. Ficha de Informações de Segurança de Produtos Químicos (FISPQ): agita $10 \mathrm{WG}$. Ribeirão Preto, 2001. 5 p.

ORGANIZATION FOR ECONOMIC COOPERATION AND DEVELOPMENT OECD. Lemna sp. growth inhibition test. In: Guideline for Testing of Chemicals. 2006. 22 p.

PRESTES, E.B.; CLEMENTE CASTRO, V.L.S.; JONSSON, C.M. Avaliação da Toxicidade Aguda de Piraclostrobin, Epoxiconazole sua Mistura em Colossoma macropomum (tambaqui). Ecotoxicology and Environmental Contamination, v. 8, n. 1, p. 125-128, 2013.

PRESTES, E.B.; JONSSON, C.M. Castro VLSS Avaliação da toxicidade de piraclostrobin, epoxiconazol e sua mistura em alga Pseudokirchneriella subcapitata. Pesticidas: Revista de Ecotoxicologia e Meio Ambiente, v. 21, p. 39-46, 2011.

ROBINSON, A.A.; BELDEN, J.B.; LYDY, M.J. Toxicity of fluoroquinolone antibiotics to aquatic organisms. Environmental Toxicology and Chemistry, v. 24, n. 2, p. 423-430, 2005.

ROWLAND, S.J.; MIFSUD, C.; NIXON, M.; READ, P.; LANDOS, M. Use of formalin and copper to control ichthyophthiriosis in the Australian freshwater fish silver perch (Bidyanus bidyanus Mitchell). Aquaculture Research, v. 40, n. 1, p. 44-54, 2008.

SCHERING-PLOUGH ANIMAL HEALTH. Aquaflor: Florfenicol: technical monograph for catfish health professionals. 2009. 36 p.

SCHMAHL, G.; MEHLHORN, H. Treatment of fish parasites 2. Effects of sym. Triazinona (toltrazuril) on monogenea. Parasitology Research, v. 75 , p. $132-145,1988$.

SCHMAHL, G.; MEHLHORN, H.; TARACHEWSKI, H. Treatment of fish parasites: Effects of sym. triazinone (toltrazuril) on skin parasitic Ciliophora (Ichthyophthirius multifilis FOUQUET 1876, Glossatella amoebae GRENFELL 1884, and Trichodina spp. (EHRENBERG, 1831). European Journal of Protistology, v. 24, p. 152-161, 1989.

SELJESTOKKEN, B.; BERGH, O.; MELINGEN, G.O.; RUDRA, H.; HETLILIDOLSEN, R.; SAMUELSEN, O.B. Treating experimentally induced vibriosis (Listonella anguillarum) in cod, Gadus morhua L., with florfenicol. Journal of Fish Diseases, v. 29, p. 737-742, 2006.

SYNGENTA. Ficha de Informação de Segurança de Produtos Químicos (FISPQ): CRUISER 700WF. 2006. 7 p.

TOMIZAWA, M.; CASIDA, J.E. Structure and diversity of insect nicotinic acetylcholine receptors. Pest Management Science, v. 57, p. 914-922, 2001.

TREVIS, D.; HABR, S.F.; VAROLI, F.M.; BERNARDI, M.M. Toxicidade aguda do praguicida organofosforado diclorvos e da associação com o piretróide deltametrina em Danio rerio e Hyphessobrycon bifasciatus. Bol Inst Pesca, v. 36, n. 1, p. 53-59, 2010

U.S. OFFICE OF THE FEDERAL REGISTER USOFR. Code of Federal Regulations: title 21: food and drugs. Washington, D.C, 2015. Disponível 
em: <http://www.accessdata.fda.gov/scripts/ cdrh/cfdocs/cfcfr/CFRSearch.cfm?fr=558.261>. Acesso em: 10 fev. 2016.

VENTURINI, F.P.; CRUZ, C.; PITELLI, R.A. Toxicidade aguda do sulfato de cobre e do extrato aquoso de folhas secas de nim para o caramujo
(Pomacea canaliculata). Acta Scientiarum. Biological Sciences, v. 30, n. 2, p. 179-183, 2008. ZAGATTO, P.A.; BERTOLETTI, E. Ecotoxicologia Aquática: princípios e aplicações. São Carlos: Editora Rima, 2006. 478 p. 